Title

\title{
The role of soluble urokinase-type Plasminogen Activator Receptor (suPAR) in Chronic Kidney Disease
}

Authors:
Ade Yonata, Ian Effendi, Zulkhair Ali, Novadian Suhaimi, Suprapti
Nephrology and Hypertension Division, Internal Medicine Department
Faculty of Medicine Universitas Sriwijaya
Mohammad Hoesin Hospital - Palembang
Editor:
Rully M. A. Roesli

Received 3 April 2018, revised 31 August 2018, accepted 15 October 2018, published 1 December 2018

\begin{abstract}
Kidney disease affects 800 million children and adults worldwide, and the numbers keep increasing. A better understanding of the pathogenesis in kidney diseases, especially on a biomolecular level, is much needed to identify novel biomarkers and therapeutic targets for kidney diseases. The glomerular filtration barrier comprises endothelial cells, the glomerular basement membrane, and podocytes. The podocyte has a central role in part of the glomerular filtration barrier. The normal functioning of podocytes is particularly important in preventing the heavy proteinuria seen in nephrotic syndrome or diabetic nephropathy, or in the disease process of focal segmental glomerulosclerosis. The podocyte is injured by circulating factors, which finally results in deranged podocyte motility. Soluble urokinase-type plasminogen activator receptor (suPAR) is a circulating form of glycosyl-phosphatidylinositol uPAR domain membrane protein and is known to play a role in the pathogenesis in kidney diseases, specifically focal segmental glomerulosclerosis and diabetic nephropathy. suPAR binds to $\alpha v \beta 3$ integrin on podocyte foot processes and causes podocyte structure disorganization leading to glomerular filtration disruption and hence proteinuria. suPAR is also a potential biomarker to predict the incidence of CKD.
\end{abstract}

Keywords: FSGS, chronic kidney disease, podocyte, diabetic nephropathy, suPAR
Corresponding author:

adeyumi@gmail.com (Yonata A)

telp: +6281283165458

\section{Introduction}

Chronic kidney disease (CKD) affects 800 million children and adults all over the world. Its prevalence keeps increasing and impacted approximately $8-16 \%$ of the world's population. Patients with CKD are at a high risk of acquiring cardiovascular diseases (CVD) and death. Therefore it is important to identify high-risk patients and give proper treatment for the underlying diseases to prevent future deterioration. This renders an understanding of the pathogenesis of CKD, especially on a biomolecular level, in order to identify novel biomarkers and therapeutic targets. ${ }^{1}$

One of the proteins being investigated on its role in the pathogenesis of CKD is soluble urokinase-type plasminogen activator receptor (suPAR). The protein is a form of circulating membrane of the glycosyl-phosphatidylinositol uPAR domain. This protein is known to be involved in the pathogenesis of kidney diseases, especially focal segmental glomerulosclerosis (FSGS) and diabetic nephropathy (DN), through interaction with glomerular podocytes. High level of suPAR affects podocytes in the glomerulus causing malformation and structural abnormalities. This article aims to review and elaborate on the latest findings and development of the role of suPAR in CKD pathogenesis and its potential as a biomarker and therapy target in CKD. ${ }^{2-6}$ 


\section{suPAR Protein}

Soluble urokinase-type plasminogen activator receptor (suPAR) is a $20-55 \mathrm{kD}$ protein and is a form of circulating uPAR. uPAR itself is a membrane-bound receptor for UPA and urokinase. Until this present day, there are three known isoforms on humans and mice: the isoforms 1,2 , and 3. suPAR is derived from immature myeloid cells and its level increases in chronic infection (such as HIV), septicemia, and malignancy. uPAR is bound to the cell membrane through a form of glycosylphosphatidylinositol anchor. Once the UPAR is released from the cell membrane, it circulates in a soluble form (suPAR) and binds to the kidney podocyte's $\alpha v \beta 3$ integrin. ${ }^{2,7-8}$

\section{suPAR in Focal Segmental Glomerulosclerosis and Diabetic Nephropathy}

Focal segmental glomerulosclerosis (FSGS) is the most common histologic finding in adults with nephrotic syndrome in the US. It is known to be caused by podocyte damage. FSGS is marked morphologically by segmental sclerosis in multiple glomeruli and is clinically marked by proteinuria. FSGS can recur in the newly transplanted kidney with a $30 \%$ chance in adults and even higher in children. ${ }^{2,9}$

Clinical observations showed FSGS often develop as a de novo disease in kidney donor recipient, and happens immediately after transplantation procedure. To an extent, this leads to the speculation of a circulating etiological factor. Wei et al elucidated in Nature Medicine about an important advancement in FSGS study where there is a presence of a circulating factor involved in the development of FSGS in human and mice, namely suPAR. ${ }^{9-10}$

suPAR is formed by multiple immune cells after the cleaving of GPI anchors by cytokines such as TNF- $\alpha$. suPAR enters the glomerulus and binds and activates $\beta 3$ integrin, one of the main proteins connecting podocyte to the glomerular basal membrane (GBM). TNF- $\alpha$ can also directly activate podocyte $\alpha v \beta 3$ integrin and vinculin. Other serum factors can lower SMPDL3b in podocytes, resulting in the activation of $\alpha v \beta 3$ through binding of integrin $\alpha v \beta 3$-suPAR. Cdc42 and Rac1 can be activated by the signaling of $\alpha v \beta 3$-suPAr, and the actin cytoskeleton of the podocyte will transform from its static form into its motile/active form. The increase of podocyte $\alpha v \beta 3$ integrin activity will increase the dynamic process of the podocyte foot process, dysregulating the form and filtrating function of the glomerulus, and could manifest as proteinuria. ${ }^{11-12}$

Diabetic nephropathy (DN) is the leading cause of end- stage renal disease (ESRD) in Indonesia. DN is usually manifested as glomerular hyperfiltration and microalbuminuria; it then progresses as a glomerular filtration rate disruption and results in ESRD. suPAR has been found to have a role not only in FSGS but also in diabetic kidney diseases, whereas the increase of suPAR affects the glomerular podocyte through the activation of integrin $\alpha v \beta 3$. The activation on the diabetic patient's podocyte then changes the podocyte structures and causes proteinuria. The remaining question is the molecular mechanism of which the hyperglycaemic condition may repress or upregulate the expression of suPAR. ${ }^{5}$

\section{suPAR as a Predictor Biomarker in Kidney Disease}

High suPAR concentration level apparently can predict the incidence of CKD in diabetic patients. In a study where the population had multiple risk factors for type 2 diabetes, high level of suPAR in healthy people with predisposing factors of DM can predict the emergence of microalbuminuria, which is an early sign of diabetic nephropathy, years before microalbuminuria appears. Since microalbuminuria is an important predictor for GFR decline, suPAR has an excellent potential in predicting diabetic nephropathy. ${ }^{13-14}$

A study by Hayek et al in 2015 resulted in a breakthrough finding in nephrology, which stated suPAR as a novel potential biomarker for predicting kidney damage progression for CKD a few years in advance from the onset of the disease. Hayek et al found that patients with the higher suPAR level have lower eGFR and higher proteinuria. Risk of CKD is three-fold higher for subjects in the highest quartile of suPAR level. For subjects with very high suPAR level (over $4020 \mathrm{pg}$ / $\mathrm{mL}$ ), the incidence risk for CKD in 10 years was found in $80 \%$ of the samples. ${ }^{13}$

The study by Hayek et al was done on subjects with cardiovascular diseases. A study that showed the role of suPAR as a predictor biomarker in the general population was done by Schulz et al on 5381 samples in Sweden. It was shown that participants in the quartile of highest suPAR levels have a lower mean of eGFR on follow-up compared to those in the quartile of low SUPAR. This study further proves that the role of suPAR in kidney diseases is not limited to FSGS and DN. ${ }^{3}$

\section{SuPAR as Therapeutic Target}

There are not many published studies investigating the role of suPAR as a therapeutic target, either on FSGS or DN, in humans. The latest study in animals is aimed to block the binding of $\alpha v \beta 3$ integrin ligand by a mono- 
clonal antibody bound to subunit $\beta 3$ of the $\alpha v \beta 3$ integrin. The study showed that targeting of $\alpha v \beta 3$ integrin with monoclonal antibody can improve histological changes related to DN in hyperglycaemic pigs. It was also shown to help reduce proteinuria and inhibit protein synthesis associated with DN on diabetic mice's kidneys. Sateck et al have done clinical research on humans using multimodal therapy, including plasma exchange in FSGS patients. The therapy was shown to reduce recurrence of FSGS, a decrease in suPAR levels and a decrease in $\beta 3$ integrin activity. A significant proteinuria reduction is also found with this therapy. However, this effect has been shown to be of temporary nature, with a rebound effect of an increase in suPAR level and $\beta 3$ integrin reactivation, as soon as 4 days after therapy administration. Whether a better clinical outcome will be achieved with a longer period of lower suPAR level is yet to be proven. ${ }^{14-16}$ Latest findings regarding suPAR and its association with CKD has brought to light a new understanding of the mechanisms of FSGS and DN, and increase our chances in predicting CKD.

\section{Conclusion}

suPAR is a biomolecule originating from bone marrow myelocytes, an UPAR protein released from myelocyte membranes and circulate in the blood vessels. suPAR has a role in the pathogenesis of FSGS and DN by entering the glomerulus, bound to $\alpha v \beta 3$ integrin at the podocyte foot process and changing the podocyte structures by signaling activity, disrupting glomerular filtration, and thus inducing proteinuria. Based on large observational studies, suPAR is also considered as a highly potential candidate as a novel biomarker in predicting CKD.

\section{References}

1. Hill NR, Fatoba ST, Oke JL, Hirst JA, O'Callaghan CA, Lasserson DS, et al. Global Prevalence of Chronic Kidney Disease - A Systematic Review and Meta-Analysis. PLoS One. 2016;11(7):e0158765.

2. Zeier M, Reiser J. suPAR and chronic kidney disease-a podocyte story. Pflugers Arch. 2017 Aug;469(7-8):1017-20.

3. Schulz CA, Persson M, Christensson A, Hindy G, Almgren P, Nilsson PM, et al. Soluble Urokinase-type Plasminogen Activator Receptor (suPAR) and Impaired Kidney Function in the Population-based Malmo Diet and Cancer Study. Kidney Int Rep. 2017 Mar;2(2):239-47.
4. Rosenberg AZ, Kopp JB. Focal Segmental Glomerulosclerosis. Clin J Am Soc Nephrol. 2017 Mar 7;12(3):502-17.

5. Dande RR, Peev V, Altintas MM, Reiser J. Soluble Urokinase Receptor and the Kidney Response in Diabetes Mellitus. J Diabetes Res. 2017;2017:3232848.

6. IRR. 8th Report Of Indonesian Renal Registry2015 [cited $20181 \mathrm{March}$ ]: Available from: http://www. depkes.go.id/download.php?file $=$ download/pusdatin/infodatin/infodatin $\% 20$ ginjal\%202017.pdf.

7. Hahm E, Wei C, Fernandez I, Li J, Tardi NJ, Tracy $\mathrm{M}$, et al. Bone marrow-derived immature myeloid cells are a main source of circulating suPAR contributing to proteinuric kidney disease. Nat Med. 2017 Jan;23(1):100-6.

8. Thuno M, Macho B, Eugen-Olsen J. suPAR: the molecular crystal ball. Dis Markers. 2009;27(3):15772.

9. Shankland SJ, Pollak MR. A suPAR circulating factor causes kidney disease. Nat Med. 2011 Aug 4;17(8):926-7.

10. Wei C, El Hindi S, Li J, Fornoni A, Goes N, Sageshima J, et al. Circulating urokinase receptor as a cause of focal segmental glomerulosclerosis. Nat Med. 2011 Jul 31;17(8):952-60.

11. Kronbichler A, Saleem MA, Meijers B, Shin JI. Soluble Urokinase Receptors in Focal Segmental Glomerulosclerosis: A Review on the Scientific Point of View. J Immunol Res. 2016;2016:2068691.

12. Smith HW, Marshall CJ. Regulation of cell signalling by uPAR. Nat Rev Mol Cell Biol. 2010 Jan;11(1):23-36.

13. Hayek SS, Sever S, Ko YA, Trachtman H, Awad M, Wadhwani S, et al. Soluble Urokinase Receptor and Chronic Kidney Disease. N Engl J Med. 2015 Nov 12;373(20):1916-25.

14. Maile LA, Busby WH, Gollahon KA, Flowers W, Garbacik N, Garbacik S, et al. Blocking ligand occupancy of the alphaVbeta3 integrin inhibits the development of nephropathy in diabetic pigs. Endocrinology. 2014 Dec;155(12):4665-75.

15. Staeck O, Slowinski T, Lieker I, Wu K, Rudolph B, Schmidt D, et al. Recurrent Primary Focal Segmental Glomerulosclerosis Managed With Intensified Plasma Exchange and Concomitant Monitoring of Soluble Urokinase-Type Plasminogen Activator Receptor-Mediated Podocyte beta3-integrin Activation. Transplantation. 2015 Dec;99(12):2593-7. 


\section{Yonata A, et al.}

16. Maile LA, Gollahon K, Wai C, Dunbar P, Busby W, Clemmons D. Blocking alphaVbeta3 integrin ligand occupancy inhibits the progression of albuminuria in diabetic rats. J Diabetes Res. 2014;2014:421827. 